ISSN 2215-3535

\title{
Impacto del léxico mental en la comprensión lectora en niños de nivel socioeconómico bajo
}

\author{
Impact of Mental Lexicon on Reading Comprehension \\ in Children Growing up in Poverty
}

\author{
Marina Ferroni ${ }^{1}$ \\ (1) https://orcid.org/0000-0002-1133-663X \\ ${ }^{1}$ Consejo Nacional de Investigaciones Científicas y Técnicas (CONICET), \\ Instituto de Lingüística, Universidad Nacional de La Plata, Argentina
}

Resumen. Objetivo. Analizar el impacto que las representaciones léxicas de niños hispanohablantes, que crecen en contextos de pobreza, tiene sobre la comprensión lectora. Método. Se evaluó a 61 niños en una prueba de comprensión lectora y, a partir de los resultados, se dividió la muestra total en dos grupos: un grupo de alto nivel de comprensión y otro grupo de bajo nivel. Luego, se evaluaron ambos grupos en pruebas de vocabulario (representaciones semánticas del léxico mental) y el nivel lector (representaciones ortográficas del léxico mental). Asimismo, se exploró el nivel de memoria operativa de los niños, la realización de inferencias y el procesamiento morfosintáctico de lenguaje oral. Resultados. Se obtienen resultados que otorgan evidencia sobre la incidencia del léxico mental en la comprensión de textos escritos.

Palabras clave. Comprensión lectora, léxico mental, representaciones léxicas, niños, contextos de pobreza.

Abstract. Objetive. This study analyses the impact that lexical representations of Spanish-speaking children in contexts of poverty have on the reading comprehension skills. Method. To this end, an assessment was made of the reading comprehension of 61 children. The results helped identify two groups within the sample: one with a high level of comprehension, and a second with a low level of comprehension. Each group's vocabulary (semantic representations of the mental lexicon) and reading level (orthographic representations of the mental lexicon) was then assessed. Other important skills related to comprehension were likewise explored, such as working memory, inference-making ability, and morphosyntactic processing of spoken language. Results. The results provide evidence regarding the substantial impact of mental lexicon representations on reading comprehension.

Keywords. Reading comprehension, Mental lexicon, Mental representations, Children, Poverty.

${ }^{1}$ Marina Ferroni. Consejo Nacional de Investigaciones Científicas y Técnicas (CONICET), Instituto de Lingüística, Universidad Nacional de La Plata, Argentina. Dirección postal: Ceretti 3553. Ciudad Autónoma de Buenos Aires. Argentina. Código postal 1431. E-mail: ferronimarina@gmail.com

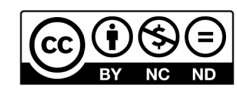

Esta obra está bajo una licencia de Creative Commons Reconocimiento-NoComercial-SinObraDerivada 4.0 Internacional. 


\section{Introducción}

La comprensión de textos resulta fundamental dado que constituye el principal mecanismo mediante el cual los niños incorporan nuevos conocimientos en el ámbito escolar (Defior, 2015). Se ha señalado que el léxico mental, el almacén que contiene información sobre las palabras conocidas, impacta de manera directa en la comprensión de textos escritos (Perfetti \& Stafura, 2014). En Argentina, una evaluación nacional para medir el nivel de comprensión lectora de niños de diferentes edades señaló una leve mejoría en el desempeño entre los años 2016 y 2018 (en 2016, 31.2 \% de niños por debajo del nivel satisfactorio y en 2018, 14.7\%), (Ministerio de Educación, Cultura, Ciencia y Tecnología, 2016, 2018). Sin embargo, los resultados obtenidos en la última medición, desagregados por sector social de procedencia, señalaron la existencia de una importante brecha entre niños de diferente sector socioeconómico a favor de los niños de sectores medios y altos (27.5\% de niños de NSE medio por debajo del nivel satisfactorio versus $42.5 \%$ en sectores de nivel socioeconómico bajo).

En este sentido, diferentes estudios realizados en este mismo país han reportado la existencia, en contextos de pobreza, de un elevado porcentaje de niños que enfrentan dificultades en su proceso de alfabetización y, por lo tanto, en el desarrollo del léxico mental y del proceso de comprensión lectora (Diuk et al., 2017; Ferroni et al., 2019). Por tanto, el presente estudio propone analizar el impacto que las representaciones léxicas de niños de escolaridad primaria que viven en contextos de pobreza urbana tiene sobre la comprensión de textos escritos.

\section{La incidencia del léxico mental en la comprensión lectora}

La comprensión lectora, es decir, la habilidad de los sujetos de construir una representación mental de un texto escrito (Ouellette \& Beers, 2010; Perfetti et al., 2005; Van den Broek, 1994) ha sido definida como una habilidad compleja en la cual intervienen innumerables procesos como el conocimiento previo, la realización de inferencias, el nivel de memoria operativa, el nivel de comprensión del lenguaje oral y otros mecanismos cognitivos de los lectores que posibilitan la conformación de una representación mental del texto escrito (Barreyro et al., 2017; Hall et al., 2019; Ouellette \& Beers, 2010; Perfetti et al., 2005; Vergara et al., 2016; Van den Broek, 1994).

Ante la complejidad del proceso, diferentes aspectos de la habilidad de comprender textos escritos han sido explicados por diferentes modelos teóricos (Perfetti \& Stafura, 2014; Swart et al., 2017). Perfetti y Stafura (2014) han propuesto un modelo de comprensión de textos escritos en el cual el léxico mental de los sujetos juega un rol fundamental. En efecto, el léxico mental es un almacén en el cual se encuentran representaciones sobre las palabras que los sujetos conocen. En estas representaciones se especifica la información sobre la forma ortográfica y fonológica de las palabras y sobre su contenido semántico (Perfetti, 2007).

Las representaciones ortográficas especifican la cadena de letras que conforman las palabras y permiten que las mismas sean reconocidas por el lector de forma automática, inconsciente y sin esfuerzo (Share, 2011). En español, una lengua ortográficamente transparente, la cantidad y calidad de representaciones ortográficas almacenadas en el léxico 
puede medirse a través de la velocidad lectora y la escritura de palabras ortográficamente complejas habilidades que constituyen el nivel lector de los sujetos (Ferroni, 2012; Signorini et al., 2001; Perfetti, 2007).

Los estudios que han explorado la relación entre el nivel lector de los niños y la medida de comprensión textual señalan que el reconocimiento automático de las palabras en la lectura permite que los recursos cognitivos de los lectores puedan ser destinados al proceso de comprensión textual (Rakhlin et al., 2019; Perfetti \& Stafura, 2015). Un proceso de decodificación lento y trabajoso, en cambio, sobrecargaría la memoria operativa afectando de forma negativa la construcción de significado del texto a partir de la lectura (Perfetti \& Hogaboam, 1975; van den Broek et al., 2017). Por otra parte, además de promover el reconocimiento automático de las palabras durante la lectura, el léxico mental actúa como una fuente que permite atribuir el contenido semántico a las palabras incluidas en los textos. En lectores expertos, una vez que las representaciones ortográficas permiten evocar las representaciones fonológicas de la palabra, el léxico otorga el significado de estas, proceso que le permite al lector construir significado mientras avanza en la lectura (Perfetti, 2007; Swart et al., 2017).

Diversos estudios realizados en diferentes lenguas han reportado evidencia de una relación estrecha entre el nivel de vocabulario y la comprensión lectora (Cain \& Oakhill, 2006; Elbro \& Buch-Iversen, 2013). En términos generales, estas investigaciones señalan que conocer el significado de las palabras que conforman los textos escritos es fundamental para establecer conexiones entre diferentes partes de estos y relacionar lo leído con el conocimiento previo del lector (Strasser et al., 2013; Swart et al., 2017).

La idea de que el léxico mental constituye un "punto de presión" en el proceso de comprensión de textos escritos ha sido ampliamente apoyada por distintos estudios que señalan que los buenos comprendedores poseen una mayor cantidad y calidad de entradas léxicas (Perfetti, 2007; Richter et al., 2013: Swart et al, 2017). Sin embargo, los diseños de los estudios sobre comprensión lectora suelen incluir sólo uno de los aspectos del léxico mental (representaciones semánticas u ortográficas de las palabras) dejando de lado la importancia de la naturaleza multidimensional de las entradas léxicas.

Por otra parte, un número importante de estudios longitudinales, que analizaron la relación entre la cantidad y calidad de representaciones en el léxico mental y la comprensión lectora, ha señalado que la velocidad en la lectura (léxico ortográfico) es la variable que mayormente se asocia a la comprensión de textos escritos en segundo y tercer grado. Sin embargo, en momentos más avanzados del desarrollo lector serían otras habilidades, como las de procesamiento de discurso oral (entre ellas el nivel de vocabulario, es decir, las representaciones semánticas) las que mayormente impactarían en la comprensión de textos escritos (Gentaz, Sprenger-Charolles, Theurel, \& Cole, 2013; Tobia \& Bonifacci, 2015).

Desde la perspectiva de los estudios longitudinales, se esperaría que las diferencias entre varios niveles de comprensión lectora en niños de quinto grado se originen en diferencias en el nivel de vocabulario más que en el nivel lector. Sin embargo, dado el patrón de desarrollo del proceso de alfabetización documentado en niños de entornos socialmente vulnerados (Diuk et al., 2017; Ferroni et al., 2019; Urquijo et al., 2015) resultaría interesante 
analizar si los resultados obtenidos en estudios con población sin carencias materiales graves se repiten en niños que crecen en contextos de pobreza.

\section{El desarrollo del proceso de alfabetización en niños que crecen en contextos de pobreza}

Un importante número de estudios que analizaron la relación entre el léxico mental y la comprensión de textos escritos han sido realizados en poblaciones sin carencias materiales graves. Sin embargo, numerosas investigaciones que comparan el desempeño lector en niños de diferentes sectores socioeconómicos han señalado que los niños que crecen en entornos de nivel socioeconómico bajo suelen presentar un menor nivel lector que niños de otros sectores sociales (Gentaz et al., 2013, Gentaz, Sprenger-Charolles, \& Theurel, 2015; Urquijo et al., 2015). Por ejemplo, en Argentina, diferentes estudios que analizaron el proceso de alfabetización en niños de entornos socioeconómicos bajos encontraron un muy bajo nivel en el desempeño en la escritura de palabras (el 28\% de los niños de tercer grado no podían escribir al dictado más de tres palabras de estructura fonológica simple) (Diuk et al., 2017) y bajos niveles de velocidad lectora (50\% de lectores lentos según baremos españoles) (Ferroni et al., 2019).

Estas diferencias se explicaron a partir de factores experienciales como la baja calidad de las propuestas educativas a las que acceden los niños estos sectores (Snow et al., 1998) o a factores relacionados con la percepción sobre estos niños que tienen las instituciones educativas a las que asisten (idea del déficit o deficit thinking en inglés, Valencia, 2010). A pesar de que existen estudios que analizan el desempeño en la escritura y la lectura de palabras, el desempeño en el procesamiento de textos escritos y las habilidades relacionadas con el mismo en este tipo de población han sido menos estudiados.

Por otra parte, existe un número limitado de estudios intergrupales que comparan el desarrollo del proceso de alfabetización entre niños de diferente sector social de procedencia; asimismo, los estudios intragrupales en niños que crecen en contextos de pobreza ha recibido menor atención (Garcia, 2015; Diuk et al., 2017). Dado que se sugiere la existencia de altos niveles de variabilidad intragrupal en el desempeño lector en niños que crecen en estos contextos (Diuk et al., 2019) resulta interesante analizar la relación entre las variables nombradas en este tipo de población. Por todo lo dicho anteriormente, el presente trabajo propone analizar el impacto del léxico mental en la comprensión de textos escritos en niños que crecen en entornos de nivel socioeconómico bajo.

\section{Método}

Para lograr el objetivo planteado, se llevó a cabo un estudio con diseño no experimental de tipo transeccional o transversal que tuvo un alcance correlacional (Hernández-Sampieri et al., 2014) ya que se propuso evaluar el grado de asociación entre diferentes habilidades (comprensión de textos escritos, vocabulario, y nivel lector, es decir, velocidad lectora y escritura ortográfica). La hipótesis del estudio apunta que un importante número de niños que crecen en contextos de pobreza experimentan dificultades en su proceso de alfabetización, en contraposición a los datos obtenidos en estudios longitudinales con niños sin carencias materiales graves. En el presente estudio se espera que las diferencias entre niños con diferente nivel de comprensión sean mayormente explicadas por el nivel lector (aunque también se esperan diferencias en el nivel de vocabulario). 


\section{Participantes}

Fueron incluidos en el estudio 61 niños (37 varones y 24 mujeres) de quinto grado (edad $M=10.6$ años; $D T=5.9)$ de un barrio de bajos recursos del conurbano bonaerense que concurrían a una escuela parroquial. Las escuelas parroquiales que se asientan en los barrios de bajos recursos dependen de los obispados de cada lugar, los cuales administran la gestión de la escuela mientras que el estado provincial otorga los fondos para los salarios docentes. Por esta razón, las familias de los niños que concurren a estas instituciones deben abonar una baja cuota mensual. Estas escuelas constituyen espacios de cohesión social para los niños y las familias en situación de vulnerabilidad social que no acceden a escuelas de gestión privada, pero ofrecen una alternativa positiva frente a las escuelas estatales que, en contextos socio-residenciales con alta prevalencia de necesidades básicas no satisfechas, mantienen ofertas educativas de muy baja calidad y climas educativos de alta conflictividad social y emocional (Tuñón \& Di Paolo, 2018).

Respecto de la medida de nivel socioeconómico de los sujetos, como es común en las sociedades latinoamericanas, las personas que viven en contextos socialmente vulnerados sufren segregación residencial (entre otras), hecho que genera la conformación de grandes áreas de viviendas precarias con bajos niveles de servicios básicos (Sabatini, 2003) que tienden a ser socialmente homogéneas (Groisman \& Suárez, 2009). En efecto, ha sido señalado en numerosas ocasiones la existencia de una importante asociación entre el lugar de residencia y el nivel socioeconómico de los sujetos (Diuk et al., 2019; Hackman \& Farah, 2009; Hanscombe et al., 2012), razón por la cual el lugar de residencia de los sujetos fue tomado en el presente estudio como indicador socioeconómico per se.

Como información adicional sobre el nivel socioeconómico de los niños incluidos en el estudio, se le pidió a los directivos del colegio información (que permanecería confidencial) sobre la ocupación de los adultos responsables de los niños. El estado ocupacional de los padres se clasificó siguiendo el de Sautu (1992) escala ocupacional, que organiza ocupaciones de acuerdo con nueve categorías. El uso de esta escala está relacionado con el hecho de que fue diseñado para incluir incluso trabajos típicos de la economía argentina. Todos los padres tenían ocupaciones clasificadas en las tres categorías inferiores de la escala. No se solicitó información sobre ingresos, porque reportar ingresos anuales no constituye una práctica habitual en Argentina en ningún nivel socioeconómico. Asimismo, en este sector social, los adultos tienden a tener trabajos informales en los que el ingreso diario sufre de un alto nivel de variabilidad, hecho que dificulta el cálculo de ganancias anuales o incluso mensuales (Diuk et al., 2019).

Respecto de la conformación de los grupos en el estudio, luego de una primera exploración de los resultados en la prueba de comprensión de textos escritos, se constituyeron dos grupos con diferente nivel de comprensión lectora: un grupo de nivel alto de comprensión $(n=19)$ y un grupo de nivel más bajo de comprensión lectora $(n=42)$. El grupo de nivel alto de comprensión fue integrado por sujetos que obtuvieron una media de comprensión de un desvío y medio sobre la media de la muestra general.

Con anterioridad al comienzo de las sesiones de evaluación, las familias de todos los niños firmaron un consentimiento informado expresando su conformidad para que estos 
participen en el proyecto de investigación. El consentimiento fue enviado desde la escuela a los hogares de los niños mediante el cuaderno de comunicaciones. Los niños participantes manifestaron su asentimiento en forma verbal. En todo el transcurso de las sesiones de evaluación, la evaluadora estuvo atenta a que no se produjera ningún tipo de malestar que justificara la exclusión de algún niño del estudio.

Asimismo, se aplicaron los procedimientos recomendados por la American Psychological Association (1992) para el trabajo con niños, los principios establecidos por la Convención Internacional sobre los Derechos del Niño y lo establecido en la Ley No 114 de Protección Integral de los Derechos de Niños, Niñas y Adolescentes de la Ciudad de Buenos Aires.

\section{Instrumentos}

Comprensión lectora. Siguiendo a Cuetos, Rodríguez, Ruan y Arribas (2014) se adaptaron dos textos narrativos (TEXTOS A y B de aquí en más) acordes a la edad escolar de los participantes de aproximadamente 300 palabras cada uno. Asimismo, se construyeron 8 preguntas (4 sobre información literal del texto y 4 sobre información que los niños debían inferir a partir de información que otorgaba el texto) con el objetivo de medir el nivel de comprensión escrita.

A la mitad de la muestra, se les presentó para su lectura el TEXTO A y a la otra mitad el TEXTO B. Luego de la lectura, se retiraban los textos narrativos y los participantes respondían las preguntas de manera escrita. Se asignó un punto a cada respuesta correcta. El alfa de Cronbach a partir de las respuestas dadas para el TEXTO A fue de .76 y para las respuestas dadas al TEXTO B del .83. No se obtuvieron diferencias estadísticamente significativas entre las respuestas correctas entre textos A y B $(t(61)$ $=1.59, p=.201)$.

Lectura de palabras. Con el fin de medir la precisión y la velocidad en la lectura de palabras, se administró la prueba PROLEC R (Cuetos et al., 2014). La prueba consta de 40 ítems de diferente longitud y nivel de complejidad silábica, los cuales eran presentados para su lectura en voz alta a los participantes. Se asignó un punto a cada palabra correctamente decodificada y se tomó el tiempo de lectura para calcular la cantidad de palabras por minuto que lograban leer correctamente los niños.

Escritura convencional. En esta prueba, por medio de un dictado, los participantes debían escribir 15 palabras con complejidad ortográfica (Ferroni, 2012).

Vocabulario. Se administró una prueba de vocabulario receptivo (Prueba de Vocabulario en Imágenes Peabody, Dunn, 1986) y una de vocabulario expresivo (K- Bit, Kaufman \& Kaufman, 1997).

Memoria. Fue evaluada mediante las pruebas de retención de dígitos de la batería WISC III (Wechsler, 1993) (dígitos en orden directo y dígitos en orden inverso).

Retención de dígitos en orden directo: se les solicitaba a los niños que escucharan con atención 8 secuencias de números para que posteriormente, las repitieran en el mismo orden en que fueron dichas. 
Retención de dígitos en orden inverso: al igual que en la prueba anterior, los niños debían oír con atención una secuencia de números, pero luego debían repetirla en el orden contrario en el que fue escuchada.

Procesamiento morfosintáctico. Se administró el sub-test "estructura de oraciones" de la prueba CELF 4 (Semel et al.,2006) que permite analizar el procesamiento y la comprensión oral de diferentes tipos de oraciones. El sub-test consta de 31 oraciones que son leídas a los niños mientras observan cuatro imágenes diferentes. Luego de escuchar la frase que lee el experimentador, el niño debe señalar la imagen que corresponde a la frase leída.

Realización de inferencias a partir de textos orales. Con el fin de medir las habilidades inferenciales de los participantes, la evaluadora leía pequeños textos narrativos a los niños. Luego de terminada la lectura, los participantes respondían de manera oral 4 preguntas sobre información que había que inferir. Se asignaba un punto a cada respuesta correcta. Se obtuvo un estadístico de Cronbach de .89 para esta medida.

\section{Procedimientos}

La prueba de comprensión lectora fue administrada grupalmente en la escuela y en el aula en la cual los niños realizan sus tareas escolares diariamente. Las demás pruebas fueron administradas en dos sesiones individuales de evaluación de aproximadamente 20 minutos cada una. La toma de datos se llevó a cabo entre los meses de octubre y noviembre, es decir, concluyendo el año escolar.

\section{Estrategias de análisis}

Con el propósito de comparar las medias obtenidas entre los grupos con diferente nivel de comprensión lectora, se analizaron, en primer lugar, las distribuciones de los resultados obtenidos de las pruebas empleadas: comprensión de textos escritos, generación de inferencias, vocabulario receptivo y productivo, precisión lectora, velocidad lectora, dígitos directo, dígitos inverso, escritura ortográfica y procesamiento morfosintáctico. En segundo lugar, se llevó a cabo un análisis multivariante de varianza (MANOVA), introduciendo la variable grupo (de diferente nivel de comprensión textual) como factor y a todas las variables evaluadas como dependientes.

\section{Resultados}

En primer lugar, se analizaron las distribuciones de las puntuaciones obtenidas en las tareas administradas. Este análisis permitió observar que las medidas de repetición de dígitos (directo) $(Z$ de Kolmogorov-Smirnov $=.252, p<.000)$, de dígitos inverso $(Z$ de Kolmogorov-Smirnov $=.215, p<.000)$, vocabulario receptivo $(Z$ de KolmogorovSmirnov $=.271, p=.044)$ y precisión lectora $(Z$ de Kolmogorov-Smirnov $=.271, p=$ .000) se alejaron significativamente de la distribución normal asintótica.

A partir de este análisis se realizó una normalización de las medidas que mostraron un alejamiento significativo a los percentiles teóricos de la distribución normal, mediante su transformación al logaritmo natural y se llevó a cabo un ANOVA de una vía con "nivel de comprensión" como factor y todas las habilidades evaluadas como variables dependientes con el fin de explorar si existieron diferencias entre grupos en las medidas 
obtenidas. Dado que los resultados obtenidos de las variables transformadas no difieren de las variables no transformadas, se muestran los estadísticos descriptivos de las mismas pruebas sin transformación. La Tabla 1 muestra los resultados obtenidos.

El análisis de los datos permitió observar la existencia de diferencias significativas entre grupos (en todos los casos a favor del grupo de mayor nivel de comprensión lectora) en las medidas de comprensión lectora $\left(F_{(1,60)}=93.18, p=.001\right)$, de comprensión de información literal del texto $\left(F_{(1,60)}=25.13, p=.001\right)$, de generación de inferencias a partir del texto escrito $\left(F_{(1,60)}=23.11, p=.001\right)$, de velocidad en la lectura de palabras $\left(F_{(1,60)}=\right.$ $7.61, p=.002)$, de escritura ortográfica $\left(F_{(1,60)}=8.72, p=.005\right)$ y de vocabulario expresivo $\left(F_{(1,60)}=10.21, p=.002\right)$.

Por otra parte, con el fin de analizar la relación existente entre las variables incluidas en el estudio, se realizaron correlaciones (Pearson) entre las habilidades evaluadas en cada uno de los grupos. Los resultados se muestran en la Tabla 2 y Tabla 3.

Tabla 1

Estadísticos descriptivos de las habilidades evaluadas en ambos grupos

\begin{tabular}{|c|c|c|c|c|c|}
\hline & \multicolumn{2}{|c|}{$\begin{array}{l}\text { Grupo de alto nivel } \\
\text { de comprensión }\end{array}$} & \multicolumn{2}{|c|}{$\begin{array}{l}\text { Grupo de medio y bajo } \\
\text { nivel de comprensión }\end{array}$} & \multirow[b]{2}{*}{ Sig. } \\
\hline & $M$ & $D T$ & $M$ & $D T$ & \\
\hline Comprensión lectora & 6.47 & 1.58 & 3.59 & 2.04 & .000 \\
\hline Respuestas literales & 3.47 & .84 & 2.09 & 1.05 & .000 \\
\hline Respuestas inferenciales & 3.00 & .74 & 1.50 & .99 & .000 \\
\hline \multicolumn{6}{|l|}{ Lectura de palabras } \\
\hline Velocidad lectora (pal. x min.) & 58.79 & 19.80 & 42.30 & 22.35 & .008 \\
\hline Precisión lectora & 38.31 & 1.63 & 35.88 & 6.42 & .110 \\
\hline Escritura ortográfica & 11.20 & 2.75 & 8.59 & 1.27 & .005 \\
\hline \multicolumn{6}{|l|}{ Vocabulario } \\
\hline Expresivo & 12.89 & 2.99 & 9.54 & 4.08 & .002 \\
\hline Receptivo & 89.55 & 14.15 & 86.07 & 12.63 & .349 \\
\hline \multicolumn{6}{|l|}{ Memoria } \\
\hline Dígitos directo & 6.63 & 1.38 & 6.16 & 1.20 & .189 \\
\hline Dígitos inverso & 5.78 & 1.39 & 5.26 & 1.03 & .105 \\
\hline $\begin{array}{l}\text { Procesamiento morfosintáctico } \\
\text { de lenguaje oral }\end{array}$ & 27.94 & 2.12 & 26.8 & 2.50 & .112 \\
\hline Inferencias sobre texto oral & 3.05 & .77 & 2.78 & 1.07 & .334 \\
\hline
\end{tabular}




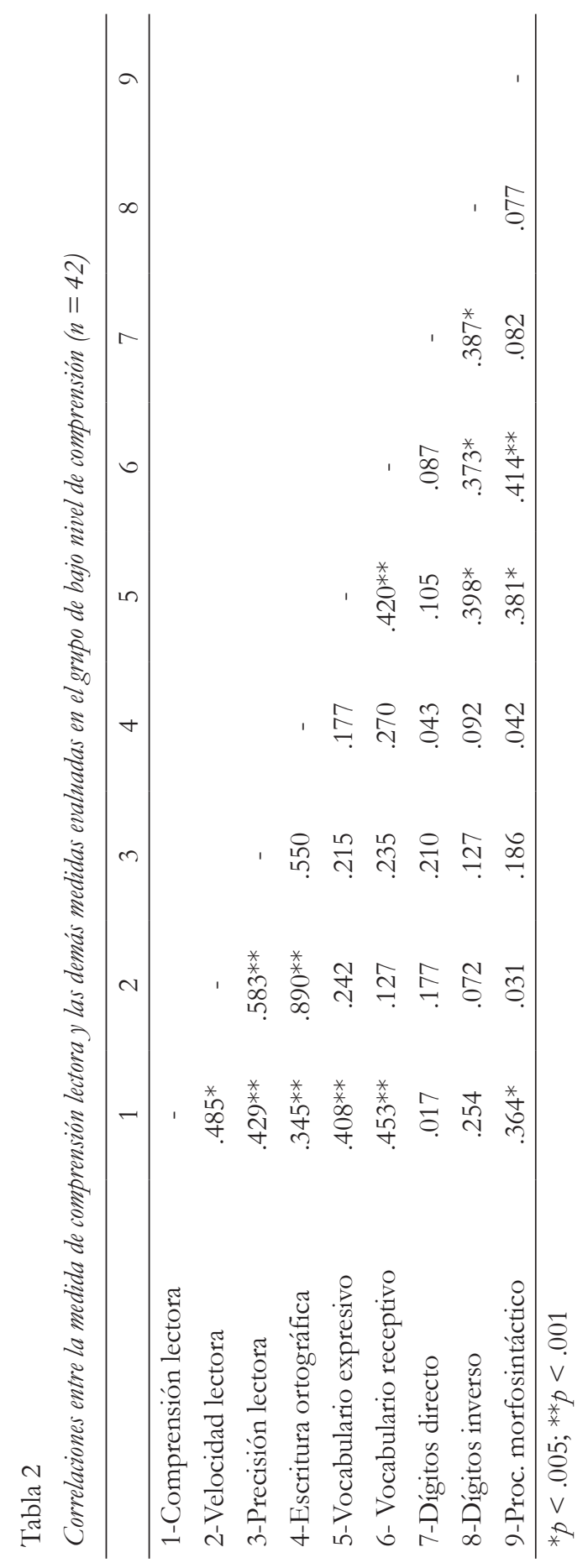

Actualidades en Psicología, 34(129), 2020, 1-16 


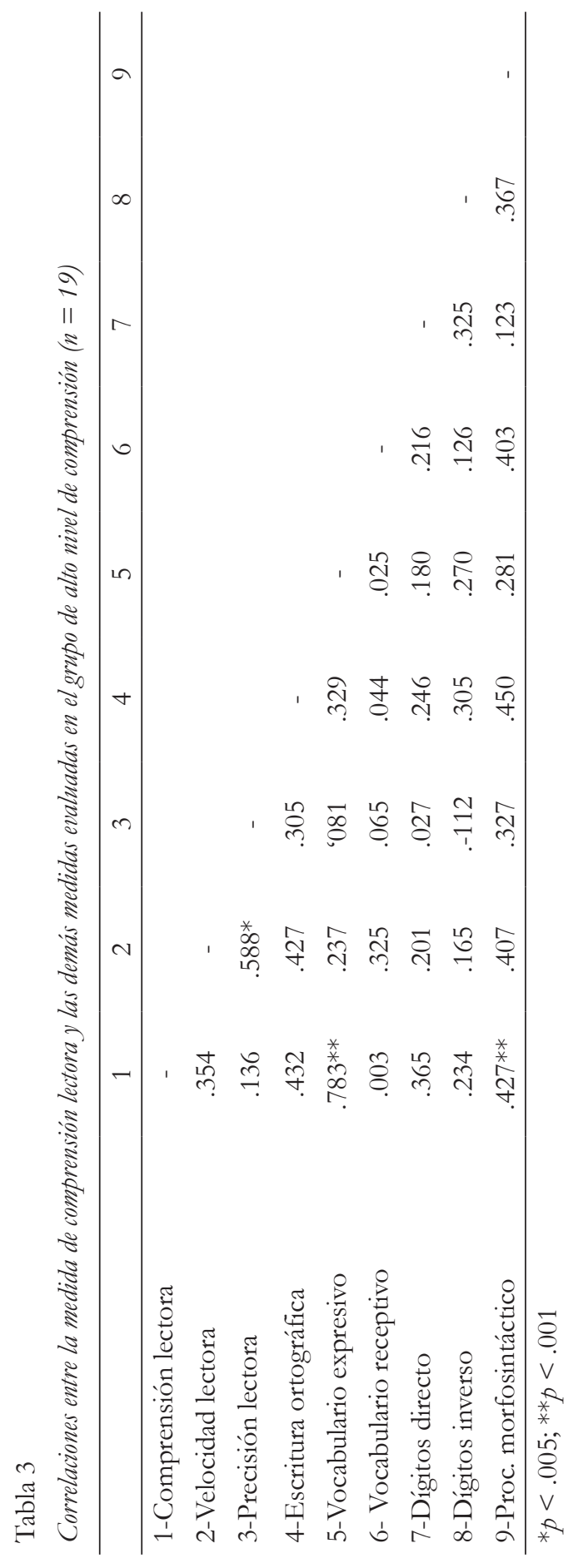

Actualidades en Psicología, 34(129), 2020, 1-16 
El análisis señaló la existencia de correlaciones significativas altas entre la medida de comprensión lectora y la medida de vocabulario y de procesamiento de lenguaje oral, en el grupo de alto nivel de comprensión. En el grupo de bajo nivel de comprensión lectora, se obtuvieron correlaciones significativas medias entre el procesamiento del texto escrito y el nivel lector a de los sujetos.

\section{Discusión}

El presente estudio explora el impacto del léxico mental en la comprensión de textos escritos en niños que crecen en contextos de pobreza. Con tal fin, se evaluó a 61 niños de barrios pobres del conurbano bonaerense en una prueba de comprensión lectora. A partir de los resultados obtenidos en dicha prueba, la muestra total fue dividida en dos grupos: un grupo de alto nivel de comprensión y otro grupo con un nivel de comprensión más bajo. Se comparó a los grupos en el nivel de vocabulario y el nivel lector. Asimismo, se exploraron otras habilidades, que la literatura sobre el tema relaciona de forma estrecha con la comprensión lectora, como la memoria operativa, la realización de inferencias y el procesamiento morfosintáctico de lenguaje oral. En términos generales, los resultados obtenidos en el presente estudio otorgan evidencia sobre la fuerte incidencia de las representaciones del léxico mental en la comprensión de textos escritos.

En la comparación de desempeño entre grupos, los datos señalan que los niños del grupo con mayor nivel de comprensión lectora habían logrado desarrollar mayor velocidad en la lectura que los niños del grupo de menor nivel de comprensión. La mayor cantidad y calidad de representaciones ortográficas de las palabras del grupo de alto nivel de comprensión lectora (las cuales permiten el reconocimiento automático en la lectura) se vio confirmada por la medida de escritura ortográfica, la cual también se diferenció entre grupos (a favor del grupo de mayor nivel de comprensión). En este sentido, los resultados concuerdan con datos presentados en otras investigaciones (Caravolas et al., 2019; Kim, 2017; Martins \& Capellini, 2018; Snow, 2018). Por otra parte, se observó que los niños del grupo de mayor nivel de comprensión tenían un mayor nivel de vocabulario que los niños del otro grupo. Este dato no resulta sorprendente dado que, como se ha señalado en numerosas investigaciones, el contenido semántico de los ítems léxicos que se reconocen durante la lectura constituye la base a partir de la cual se construyen unidades de significado más amplias (Strasser et al., 2013; Swart et al., 2017). En este sentido, los resultados obtenidos en el presente estudio concuerdan con los datos existentes en la bibliografía sobre el tema.

También, en el presente estudio se obtuvieron resultados que no concuerdan con los datos obtenidos en otras investigaciones. En efecto, los estudios longitudinales señalan que el impacto de la velocidad lectora es muy fuerte en lectores principiantes y en niños con nivel de escolaridad idéntico al de los participantes del presente estudio (Gentaz et al., 2013; Tobia \& Bonifacci, 2015), pero que decrece a medida que los niños avanzan en su proceso de alfabetización. Por ejemplo, Tobía y Bonifacini (2015) evaluaron a niños italianos (una lengua de ortografía transparente similar al español) de quinto grado mediante pruebas de comprensión lectora, habilidades de lenguaje oral y nivel lector y los resultados mostraron que las habilidades de vocabulario y procesamiento sintáctico fueron las que mayor impacto tuvieron sobre la comprensión lectora. Gentaz et al., (2013) encontraron una 
fuerte incidencia del reconocimiento de palabras en la comprensión de textos en lectores iniciales de francés, pero señalaron que el nivel de impacto iba decreciendo a medida que los niños avanzaban en su proceso de alfabetización.

Los resultados de las correlaciones realizadas en el presente estudio mostraron que el grupo de alto nivel de comprensión posee un patrón de desempeño similar al de los niños de otros estudios (Caravolas et al., 2019; Martins \& Capellini, 2018). En efecto, en este grupo la medida de comprensión textual se vio mayormente asociada a las habilidades de vocabulario y de procesamiento de lenguaje oral. Sin embargo, en el grupo de niños con bajo nivel de comprensión lectora (el 69\% de la muestra total de niño), el procesamiento textual se vio mayormente asociado a la medida de lectura de palabras. En este sentido, estos resultados concuerdan con la hipótesis planteada en este trabajo, la cual afirma que en niños de entornos sociales vulnerados, los cuales experimentan dificultades en su proceso de alfabetización, el nivel lector poseería una importante incidencia (por sobre las habilidades de procesamiento de lenguaje oral, como el vocabulario) sobre la comprensión de textos escritos aun en edades avanzadas.

Los resultados obtenidos en el presente trabajo poseen importantes implicancias pedagógicas. En efecto, a partir de los resultados obtenidos en este y otros estudios (Diuk et al., 2019; Ferroni et al., 2019) resulta fundamental analizar las variables que inciden en contenidos educativos tan importantes como la comprensión de textos escritos en poblaciones que no sean las mayoritariamente descritas en la bibliografía sobre el tema. Como resultado, investigaciones realizadas con niños de idéntica edad que los niños del presente estudio, que crecen en entornos sin carencias materiales graves, suelen señalar que los diferentes niveles de comprensión lectora se explican a partir de distintos niveles de habilidades metacogntitivas de monitoreo de la comprensión o a diferentes niveles de procesamiento morfosintáctico del lenguaje oral. Habilidades que son tenidas en cuenta a la hora de diseñar intervenciones educativas específicas. Sin embargo, existe en Argentina, un $48 \%$ que crecen en contextos con carencias materiales graves. Los resultados arrojados en el presente estudio parecen indicar que las variables que explican los diferentes niveles de comprensión lectora no son las mismas que las que arroja la investigación realizada con población mayoritaria.

Respecto de las limitaciones del estudio, resultaría necesario realizar investigaciones con esta población con mayor número de sujetos y diferentes momentos del proceso de alfabetización de los niños, con el fin de determinar el impacto de cada variable sobre la comprensión lectora en diferentes momentos del desarrollo.

\section{Referencias}

Barreyro, J., Injoque-Ricle, I., Álvarez-Drexler, A., Formoso, J., \& Burín, D. (2017). Explanation-based inferences generation in expository texts comprehension: The role of working memory and specific prior knowledge. Summa Psicológica, 24, 17-24. https://doi.org/10.1016/j.sumpsi.2016.09.002

Cain, K., \& Oakhill, J. (2006). Profiles of children with specific reading comprehension difficulties. British Journal of Educational Psychology, 76, 683-696. https://doi. org/10.1348/000709905X67610 
Caravolas, M., Lervåg, A., Mikulajová, M., Defior, S., Seidlová-Málková, G., \& Hulme, Ch. (2019). A Cross-Linguistic, Longitudinal Study of the Foundations of Decoding and Reading Comprehension Ability. Scientific Studies of Reading, 23, 386-402. https://doi.org/10.1080/10888438.2019.1580284

Cuetos, F., Rodríguez, B., Ruan, E., \& Arribas, D. (2014). PROLEC-R: Batería de evaluación de los procesos lectores, revisada ( $5^{\mathrm{a}} \mathrm{ed}$.). TEA Ediciones.

Defior, S., Serrano, F., \& Gutiérrez, N. (2015). Dificultades específicas de aprendizaje. Editorial Síntesis.

Diuk, B., Ferroni, M., Mena, M., \& Barreyro, J. (2017). Respuesta a la intervención y escritura en niños de grupos sociales vulnerados. Páginas de Educación, 10(2), 96110. https://doi.org/10.22235/pe.v10i2.1426

Diuk, B., Barreyro, J. P., Ferroni, M., Mena, M., \& Serrano, F. (2019) Reading Difficulties in Low-SES Children: A Study of Cognitive Profiles. Journal of Cognition and Development, 20, 75-95. doi:10.1080/15248372.2018.1545656

Dunn, L. M. (1986). Test de vocabulario en imágenes Peabody. MEPSA.

Elbro, C., \& Buch-Iversen, I. (2013). Activation of Background Knowledge for Inference Making: Effects on Reading Comprehension. Scientific Studies of Reading, 17, 435452. https://doi.org/10.1080/10888438.2013.774005

Ferroni, M. (2012). La adquisición de conocimiento ortográfico en español [Tesis no publicada para optar por el grado de Doctora]. Universidad Nacional de La Plata. Facultad de Humanidades y Ciencias de la Educación: Buenos Aires.

Ferroni, M., Barreyro, J. P., Mena, M., \& Diuk, B. (2019). Perfiles cognitivos de niños de nivel socioeconómico bajo con dificultades en la velocidad lectora: análisis de los resultados de una intervención. Interdisciplinaria, 36(1), 273-288.

Garcia, C. C. (2015). Editorial: Continuity and change in child development. Child Development, 86, 7-9. https://doi.org/10.1111/cdev.12353

Gentaz, E., Sprenger-Charolles, L., \& Theurel, A. (2015). Differences in the predictors of reading comprehension in first graders from low socio-economic status families with either good or poor decoding skills, Plos One, 10(3), e0119581. https://doi. org/10.1371/journal.pone.0119581

Gentaz, E., Sprenger-Charolles, L., Theurel, A., \& Colé, P. (2013). Reading comprehension in a large cohort of French first graders from low socio-economic status families: A 7-month longitudinal study. PLOS ONE, 8(11), e786082. https://doi. org/10.1371/journal.pone.0078608

Groisman, F., \& Suárez, L. (2009). Residential segregation in Greater Buenos Aires. En B. Roberts y R. Wilson (Eds.), Urban segregation and governance in the Americas (pp. 39-64). Palgrave-Macmillan.

Hackman, D. A., \& Farah, M. J. (2009). Socioeconomic status and the developing brain. Trends in Cognitive Sciences, 13, 65-73. https://doi.org/10.1016/j.tics.2008.11.003 
Hall, C., Vaughn, S., Barnes, M. A., Stewart, A., Austin, C. R., \& Roberts, G. (2019). The Effects of Inference Instruction on the Reading Comprehension of English Learners with Reading Comprehension Difficulties. Remedial and Special Education, 40, 19-37. https://doi.org/10.1177/0741932518824983

Hanscombe, K. B., Trzaskowski, M., Haworth, C. M., Davis, O. S., Dale, P. S., \& Plomin, R. (2012). Socioeconomic status (SES) and children's intelligence (IQ): In a UKrepresentative sample SES moderates the environmental, not genetic, effect on IQ. PLOS One, 7(2). https://doi.org/10.1371/ journal.pone.0030320

Hernández, R., Fernández, C., \& Baptista, P. (2014). Metodología de la investigación. McGraw-Hill.

Kaufman, A. S., \& Kaufman, N. L. (1997). Test breve de inteligencia de Kaufman. TEA Ediciones.

Kim, Y. (2017). Why the Simple View of Reading Is Not Simplistic: Unpacking component skills of reading using a direct and indirect effect model of reading (DIER), Scientific Studies of Reading, 21, 310-333. https://doi.org/10.1080/10888438.2017.1291643

Martins, M., \& Cappellini, S. (2018). Relation between oral reading fluency and reading comprehension. CoDAS, 31(1). https://doi.org/10.1590/2317$1782 / 20182018244$

Ministerio de Educación, Cultura, Ciencia y Tecnología. (2016). Aprender 2016: Informe de resultados de Buenos Aires. Argentina.gob.ar https://www.argentina.gob.ar/ sites/default/files/buenos-aires-aprender-informe-final-grados-censales592c2bcc2a005_0.pdf

Ministerio de Educación, Cultura, Ciencia y Tecnología. (2018). Aprender 2018: Informe de resultados de Buenos Aires. Argentina.gob.ar https://www.argentina.gob.ar/ sites/default/files/buenos-aires-aprender-informe-final-grados-censales592c2bcc2a005_0.pdf

Ouellette, G., \& Beers, A. (2010). A not-so-simple view of reading: how oral vocabulary and visual-word recognition complicate the story. Reading and Writing, 23, 189208. https://doi.org/10.1007/s11145-008-9159-1

Perfetti, C. (2007). Reading ability: Lexical quality to comprehension. Scientific Studies of Reading, 11, 357-383. https://doi.org/10.1080/10888430701530730.

Perfetti, C. A., \& Hogaboam, T. (1975). Relationship between single word decoding and reading comprehension skill. Journal of Educational Psychology, 461-469. http:// dx.doi.org/10.1037/h0077013

Perfetti, C. A., Landi, N., \& Oakhill, J. (2005). The Acquisition of Reading Comprehension Skill. En M. J. Snowling, \& C. Hulme (Eds.), The Science of Reading: A Handbook (pp. 227-247). Blackwell.

Perfetti, C., \& Stafura, J. (2014). Word knowledge in a theory of reading comprehension. Scientific Studies of Reading, 18, 22-37. https://doi.org/10.1080/10888438.2013.827687

Perfetti, C. A., \& Stafura, J. Z. (2015). Comprehending implicit meanings in text without making inferences. En E. J. O’Brien, A. E. Cook, \& R. F. Lorch (Eds.), Inferences 
during reading (pp. 1-18). Cambridge University Press. https://doi.org/10.1017/ CBO9781107279186.002

Rakhlin, N., Mourgues, C., Cardoso-Martins, C., Korneve, A., \& Grigorenko, C. (2019). Orthographic processing is a key predictor of reading fluency in good and poor readers in a transparent orthography. Contemporary Educational Psychology, 56, 250261. https://doi.org/10.1016/j.cedpsych.2018.12.002

Richter, T., Isberner, M., Naumann, J., \& Neeb, Y. (2013). Lexical quality and reading comprehension in primary school children. Scientific Studies of Reading, 17, 415434. https://doi.org/10.1080/10888438.2013.764879

Sabatini, F. (2003). La segregación social del espacio en las ciudades de América Latina. Banco Interamericano de Desarrollo. http://idbdocs.iadb.org/wsdocs/getdocument. aspx?docnum $=1442235$

Sautu, R. (1992). Teoría y medición del estatus ocupacional. Escalas ocupacionales objetivas y de prestigio. Facultad de Ciencias Sociales (UBA).

Semel, E., Wiig, E. H., \& Secord, W. A. (2006). CELF 4. Clinical Evaluation of Language Fundamentals. Spanish Edition. PsychCorp.

Share, D. L. (2011). On the role of phonology in reading acquisition: The self-teaching hypothesis. En S. A. Brady, D. Braze, \& C. A. Fowler (Eds.), Explaining individual differences in reading: Theory and evidence. New directions in communication disorders research (pp. 45-68). Psychology Press.

Signorini, A., Borzone, A. M., \& Diuk, B. (2001). Del conocimiento fonológico al conocimiento ortográfico. Alcance y limitaciones de la perspectiva "fonocéntrica" en el procesamiento de palabras escritas. Lenguas Modernas, 28, 7-28.

Snow, C. (2018). Simple and Not-So-Simple Views of Reading. Remedial and Special Education, 39, 313-317. https://doi.org/10.1177/0741932518770288

Snow, C., Burns, M., \& Griffin, P. (1998). Preventing reading difficulties in young children: Committee on the prevention of reading difficulties in young children. National Research Council.

Strasser, K., del Río, F., \& Larraín, A. (2013). Profundidad y amplitud del vocabulario: ¿Cuál es su rol en la comprensión de historias en la edad pre-escolar? Estudios de Psicología, 34, 221-225. https://doi.org/10.1174/021093913806751401

Swart, N., Muijselaar, M., Steenbeek-Planting, E., Droop., P., de Jong., P., \& Verhoeven, L. (2017). Differential lexical predictors of reading comprehension in fourth graders. Reading and Writing, 30, 489-507. https://doi.org/10.1007/s11145-016-9686-0

Tobia, V., \& Bonifacci, P. (2015). The simple view of reading in a transparent orthography: The stronger role of oral comprehension. Reading and Writing: An Interdisciplinary Journal, 28, 939-957. https://doi.org/10.1007/s11145-015-9556-1

Tuñón, I., \& Di Paolo, G. (2018). Lo comportamental e institucional como factores asociados a las calificaciones escolares en Lengua y Matemática. Perfiles Educativos, 40, 86-99. https://doi.org/10.22201/iisue.24486167e.2018.162.58733 
Urquijo, S., García Coni, A., \& Fernandes, D. (2015). Relación entre aprendizaje de la lectura y nivel socioeconómico en niños argentinos. Avances en Psicología Latinoamericana, 33, 303-318. http://dx.doi.org/10.12804/ap133.02.2015.09

Valencia, R. (2010). Dismantling contemporary deficit thinking: Educational thought and practice. Routledge.

Van den Broek, P. (1994). Comprehension and memory of narrative texts: Inferences and coherence. En M. A. Gernsbacher (Ed.), Handbook of psycholinguistics (pp. 539588). Academic Press.

Van den Broek, P., Espin, C., McMaster, K., \& Helder, A. (2017). Developing reading comprehension interventions. Perspectives from theory and practice. En E. Segers y P. van den Broek (Ed.). Developmental Perspectives in Written Language and Literacy (pp. 85-101). John Benjamins. https://doi.org/10.1075/z.206.06van

Vergara, D., Strasser, K., \& del Río, M. F. (2016). Más que palabras por minuto: las otras habilidades que afectan la comprensión en $1^{\circ}$ básico. Revista Calidad en la Educación, 44, 46-67. http://dx.doi.org/10.31619/caledu.n44.19

Wechsler, D. (1993). WISC-R: Escala de Inteligencia de Wechslerpara Niños. TEA Ediciones. 